\title{
Structural Characterization of Beam Sensitive Pharmaceutical Compounds Using 3D Electron Diffraction-Micro-ED at Low Dose with Pixelated Detectors
}

Partha Pratim Das ${ }^{1}$, Alejandro Gómez Pérez ${ }^{2}$, Athanassios S. Galanis ${ }^{1}$ and Stavros Nicolopoulos ${ }^{1}$

${ }^{1}$ NanoMEGAS SPRL, Brussels, Brussels Hoofdstedelijk Gewest, Belgium, ${ }^{2}$ NanoMEGAS SPRL, Brussels, Brabant Wallon, Belgium

In recent years, pharmaceutical industry has shown a growing interest in characterization of API (active pharmaceutical ingredient) using Electron Diffraction due to inherent advantages of collecting 3D-ED data from nanometer size crystals in TEM.

The principle of acquiring TEM 3D-ED data for beam sensitive materials consists of focusing the electron beam on a nanometer size crystal at low dose conditions $(<0.01 \mathrm{e} / \AA 2 / \mathrm{sec})$, and sampling the reciprocal space in small steps (usually 1 degree tilt or less) using beam precession or using continuous rotation (with or without beam precession) of the crystal, technique also known as Micro-ED [1]; data collection can be done under cryo-conditions/at room temperature where data can be acquired using CCD cameras or new age sensitive pixelated detectors. Crystals can be searched in STEM mode avoiding beam damage caused by continuous TEM illumination. In low dose condition weak diffraction spots can be recorded thanks to the zero noise level of the pixelated detector, after the inelastic background is cut-out by the in-column energy filter. The acquired 3D-ED data can be processed to determine ab-initio unit cell, space group, and atomic positions and further least square refinement can be performed.

We have successfully characterized several known (e.g. carbamazepine, nicotinic acid etc.) and unknown pharmaceutical compounds (e.g. like, Ramelteon, Tolvaptan, Loratadine Form II, Linagliptin etc.) with different structural complexity (eg. unit cell ( > $35 \AA$ ) and/or up to 2 molecules in the asymmetric unit) $[2,3]$ using $3 \mathrm{D}-\mathrm{ED}$ data. In some cases it was possible to resolve the structure at room temperature using modern pixelated detectors (Medipix III) at low dose. In all cases structures were validated against existing X-Ray structure models if available and/or with DFT-D geometry optimizations or Rietveld refinement against available powder diffraction data. Thus, we can conclude that 3D-ED technique can be used as a powerful and useful tool for phase identification and structural characterization for any $\mathrm{nm}$ size $(50-500 \mathrm{~nm})$ crystal.

\section{References}

[1] M. Gemmi, E. Mugnaioli, T. E. Gorelik, U. Kolb, L. Palatinus, P. Boullay, S. Hovmöller, J. P. Abrahams. ACS Central Science 2019, 5(8), 1315-1329.

[2] E. van Genderen, M.T.B. Clabbers, P. Pratim Das, I. Nederlof, A. Stewart, Q. Portillo, S. Nicolopoulos, J.P. Abrahams, Acta Crystallogr. A 2016, 72, 236-242.

[3] P. P. Das, E. Mugnaioli, S. Nicolopoulos, C. Tossi, M. Gemmi, A. Galanis, G. Borodi, M. M. Pop, Org. Process Res. Dev. 2018, 22, 1365-1372. 